\title{
UCRL-JRNL-222870
}

LAW RENCE LIVERMORE N A T IO N A L LABORATORY

\section{Theory and Simulation of Warm Dense Matter Targets}

J. J. Barnard, J. Armijo, R. M. More, A. Friedman, I. Kaganovich, B. G. Logan, M. M. Marinak, G. E. Penn, A. B. Sefkow, P. Santhanam, J. S. Wurtele

July 14, 2006

Nuclear Instruments and Methods in Physics Research 
This document was prepared as an account of work sponsored by an agency of the United States Government. Neither the United States Government nor the University of California nor any of their employees, makes any warranty, express or implied, or assumes any legal liability or responsibility for the accuracy, completeness, or usefulness of any information, apparatus, product, or process disclosed, or represents that its use would not infringe privately owned rights. Reference herein to any specific commercial product, process, or service by trade name, trademark, manufacturer, or otherwise, does not necessarily constitute or imply its endorsement, recommendation, or favoring by the United States Government or the University of California. The views and opinions of authors expressed herein do not necessarily state or reflect those of the United States Government or the University of California, and shall not be used for advertising or product endorsement purposes. 


\title{
Theory and Simulation of Warm Dense Matter Targets
}

\author{
J. J. Barnard ${ }^{1}$, J. Armijo ${ }^{2}$ R. M. More', \\ A. Friedman', I. Kaganovich ${ }^{3}$, B. G. Logan ${ }^{2}$, M. M. Marinak', G. E. Penn' ${ }^{2}$, \\ A. B. Sefkow ${ }^{3}$, P. Santhanam ${ }^{2}$, J .S. Wurtele ${ }^{2}$ \\ 1. Lawrence Livermore National Laboratory, Livermore, CA 94550 USA \\ 2. Lawrence Berkeley National Laboratory, Berkeley CA 94720 \\ 3. Princeton Plasma Physics Laboratory, Princeton, NJ
}

\begin{abstract}
We present simulations and analysis of the heating of warm dense matter foils by ion beams with ion energy less than one $\mathrm{MeV}$ per nucleon to target temperatures of order one $\mathrm{eV}$. Simulations were carried out using the multi-physics radiation hydrodynamics code HYDRA and comparisons are made with analysis and the code DPC. We simulate possible targets for a proposed experiment at LBNL (the so-called Neutralized Drift Compression Experiment, NDCXII) for studies of warm dense matter. We compare the dynamics of ideally heated targets, under several assumed equation of states, exploring dynamics in the two-phase (fluid-vapor) regime.
\end{abstract}

PACS Numbers: 29.27.-a, 52.65.Cc,52.65.Cr

Keywords: Warm Dense Matter, Hydrodynamics, Equation of state, Accelerators 


\section{Introduction}

The use of ion beams to heat matter to warm dense matter (WDM) conditions has been suggested due to a number of potential advantages, such as precise control and uniformity of energy deposition, large sample sizes compared to diagnostic resolution volumes, ability to heat a wide variety of target materials (including both conductors and insulators), relatively long times to allow equilibrium conditions to be achieved, a benign environment for diagnostics, and high shot rates. One approach (adopted at GSI, for example) has been to use ion beams with stopping range much greater in distance than the focal spot radius of the ion beam. These targets are thus roughly cylindrical in geometry. Recently a collaboration of researchers [1] at LBNL, LLNL, and PPPL (the Heavy Ion Fusion Science Virtual National Laboratory HIFS VNL) has been exploring the possibility of using ions at lower energy (less than $1 \mathrm{MeV}$ per nucleon) but with shorter pulse ( $\sim 1 \mathrm{~ns})$ and higher current ( 100 A). The lower energy implies a very short range $(\sim 1$ to $100 \mu)$, much smaller than the radius of the focal spot $(\sim 1 \mathrm{~mm})$. The geometry of such targets is thus closer to being planar. The intent of this paper is to begin to show how equation of state and other transport parameters of interest will be inferred by understanding the hydrodynamics of foils as they are volumetrically heated by ion beams.

This paper is organized as follows. Section II outlines the basic requirements on a beam and target. Section III reviews the basic hydrodynamic equations and their solutions as given in ref. [2] for idealized equations of state. Section IV, illustrates by 
example some of the complexities of the physics when the equation of state becomes more realistic, and parts of the target fall into a two phase (liquid-vapor) region. Section V describes a numerical study which parametrically explores a particular target with beam intensities typical of a planned future US experiment NDCX II, to explore which parameters will be most useful for inferring an unknown equation of state from experimental measurements. Section VI discusses some of the neglected physics, such as surface tension effects, which will ultimately be necessary to include in simulations to understand some aspects of the target physics.

\section{Requirements on the beam and target}

Our basic strategy [3] for carrying out warm dense matter experiments using ion beams is to focus a low to moderate energy $(0.4-30 \mathrm{MeV})$ (but high current) ion beam onto a thin foil target. The exact energy and ion mass are chosen such that the beam enters the foil with an energy slightly higher than the Bragg peak, and exits the foil with an energy slightly lower than the Bragg peak [4]. This allows the energy deposition to be relatively uniform through the entire depth of the target, so that precise measurements to determine equation of state (EOS), or other material properties, could be carried out. In this paper, we focus on parameters of a proposed facility to be built at LBNL called NDCX II. One option for this facility is to use $2.8 \mathrm{MeV}$ singly charged $\mathrm{Li}$ ions. The conclusions we draw however are not limited to the NDCX II facility. These high current, low energy beams may require compression and focusing in a neutralized plasma to overcome beam space charge, and plans and experiments for this approach are described in refs [1,5-11]. 
In order to reach Warm Dense Matter conditions, enough energy must be supplied to the target to raise the temperature to a regime of interest. Although a precise estimate of the target temperature requires the use of a more sophisticated equation of state, to obtain an estimate, we may equate the energy density to the energy deposited divided by the volume over which the energy is deposited:

$(M / 2) n k T=\Delta E_{\text {ion }} N_{\text {ions }} /\left(\pi r_{\text {spot }}^{2} \Delta z\right)$

(for a uniform distribution of ion intensity on the focal spot). Here, $M$ is the number of degrees of freedom. For solids at relevant temperatures, $M=6$ (3 kinetic plus 3 "vibrational" degrees of freedom); and $\Delta z=\Delta E_{\text {ion }} /(\rho d E / d X) . \quad \Delta E_{\text {ion }}$ is the change in ion energy between entrance to and exit from the foil, chosen such that $d E / d X$ varies by some prescribed variance such as 5\%. Typically, the entrance and exit energy ( $E_{\text {entrance }}$ and $\left.E_{\text {exit }}\right)$ are chosen to be 1.5 times and 0.5 times, respectively, the energy of the peak in $d E / d X$. ( $d E / d X$ and $\Delta z$ have been obtained using the SRIM code; see figures 1.1 and 1.2). From figure 1.1, we see that for near-term experiments which are limited to energies of of a few $\mathrm{MeV}$ or less, only Li has the Bragg peak within the accessible range, of the three lower mass alkali metal ions that are candidates for hot plate ion sources.

Rearranging equation (1) and putting in values for lithium yields:

$k T=9.6 \mathrm{eV}\left(N_{\text {ions }} / 10^{13}\right)\left(1 \mathrm{~mm} / \mathrm{r}_{\text {spot }}\right)^{2}(d E / d X / 2 \mathrm{MeV} \mathrm{cm} / 2 / \mathrm{mg})\left(A_{\text {targ }} / 27\right)$.

In terms of total charge Q:

$k T=6.0 \mathrm{eV}(Q / 1 \mu \mathrm{C})\left(1 \mathrm{~mm} / \mathrm{r}_{\mathrm{spot}}\right)^{2}(d E / d X / 2 \mathrm{MeV} \mathrm{cm} / \mathrm{mg})\left(A_{\text {targ }} 27\right)$

Expressed in terms of the fluence per unit area, $F$

$k T=0.19 \mathrm{eV}\left(F / 1 \mathrm{~J} / \mathrm{cm}^{2}\right)\left(1 \mathrm{MeV} / E_{\text {entrance }}\right)(d E / d X / 2 \mathrm{MeV} \mathrm{cm} / \mathrm{mg})\left(A_{\text {targ }} / 27\right)$.

The fluence is defined as the energy integrated over the entire pulse. This formula uses 
the energy at foil entrance $E_{\text {entrance }}$ and $d E / d X$ at foil center. For Lithium at the Bragg Peak, $\mathrm{E}_{\text {peakr }}=1.88 \mathrm{MeV}, d E / d X=2.052 \mathrm{MeV} \mathrm{cm} / \mathrm{mg}$. The entrance to the foil is approximately at $50 \%$ higher energy, $E_{\text {entrance }}=2.82 \mathrm{MeV}$. Thus to reach $2 \mathrm{eV}$ (to allow for some robustness for the experiments) requires: $F=29.1 \mathrm{~J} / \mathrm{cm}^{2}$ of $\mathrm{Li}$ at $E_{\text {entrance }}=2.82$ $\mathrm{MeV}$. Using eqs. (1) - (4), similar estimates can be made for other experiments using K and $\mathrm{Na}$.

The basic beam requirement is thus the fluence per unit area $F$, which must be delivered to raise the target temperature to values of interest. However, a second important parameter is the pulse duration, $\Delta t$. The pulse duration must be sufficiently short relative to the cooling time scale, which for this regime is typically the hydrodynamic time scale $t_{\text {hydro }}=\Delta z / c_{s}$, such that significant cooling has not occurred. Here $c_{\mathrm{s}}$ is the sound speed (at the temperature of the heated material.) For aluminum at $1 \mathrm{eV}$ the sound speed is approximately $5 \times 10^{5} \mathrm{~cm} / \mathrm{s}$ so that for a 3.5 micron foil, the hydrodynamic timescale is about $0.7 \mathrm{~ns}$. Pulse durations significantly longer than $1 \mathrm{~ns}$ would thus not reach the desired temperature. Figure 1.2 shows the benefit of using Li for low energy experiments, since the larger range at solid density leads to longer hydrodynamic timescales. Thus for experiments at solid densities, hydro simulation codes are required to ensure that central temperatures of interest can be achieved, when beam pulse durations $\Delta t$ are roughly the same order as $t_{\text {hydro }}$.

\section{Foam targets}

Targets made of metallic foams are of interest in their own right, because of their use in 
Inertial Confinement Fusion and other applications, but also as a means of relaxing the pulse duration requirements on the beam for the purpose of exploring a wide range of parameters in the density-temperature parameter space. For a metallic foam with a mass density $10 \%$ of the solid density, the ion range $\Delta z$ increases by a factor of 10 over its value for solid density, and so the hydrodynamic time scale increases by that factor also. With foams it is much easier to be in a regime where the pulse duration $\Delta t<<t_{\text {hydro }}$. The main issue with foams is that they are inherently inhomogeneous. The timescale for homogenization is thought to be $t_{\text {uniform }} \sim n r / c_{s}$ where $\mathrm{n}$ is a number of order $3-5, r$ is the pore size and $c_{s}$ is the sound speed. Thus, for $n=4, r=100 \mathrm{~nm}$, the homogenization time would be one percent of the hydro timescale for a 40 micron $10 \%$ aluminum foam foil. Foams with $100 \mathrm{~nm}$ pore size should be obtainable, according to LLNL ICF researchers. This factor of 100 separation of timescales should be sufficient for our purposes of obtaining EOS data in the WDM regime. However, we should also note that the critical point is typically near one-third solid density, and the exact liquid-two-phase transition boundary at lower temperatures is at even higher densities, so the ability to operate up to solid density is important for the robustness of any WDM facility.

We should also note that ion stopping may be effected when the time between collisions is more than the relaxation timescale of the ion in the excited state. Measurements of ion stopping in foam could provide information regarding the fundamental science of ion stopping. 


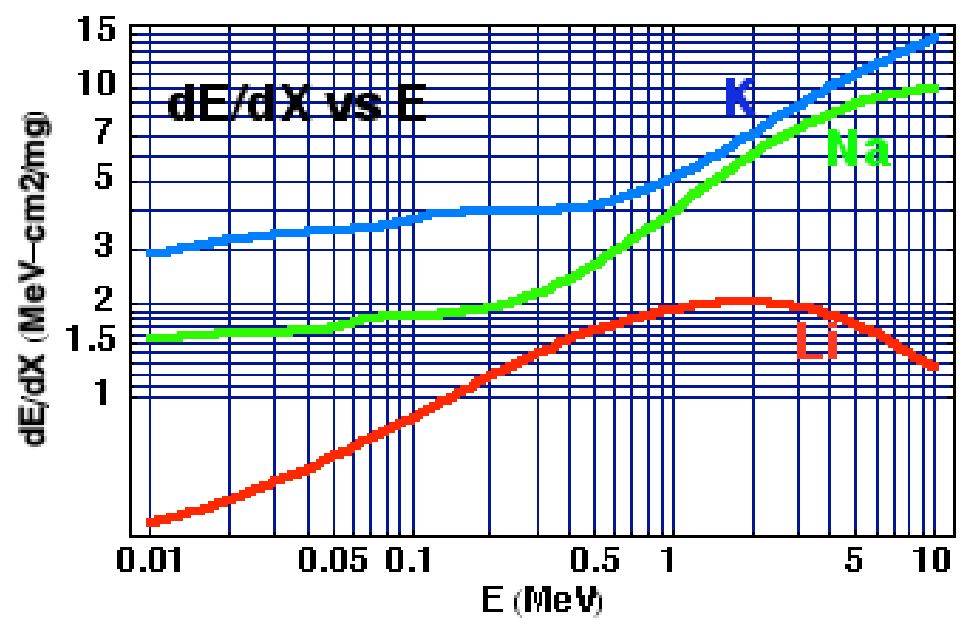

Figure 1.1 Energy loss rate $(\mathrm{dE} / \mathrm{dX})$ as a function of ion energy in solid aluminum for three different ions $(\mathrm{K}, \mathrm{Na}$, and $\mathrm{Li})$ for energies that include range of current and near-term future experiments (data from the SRIM code).

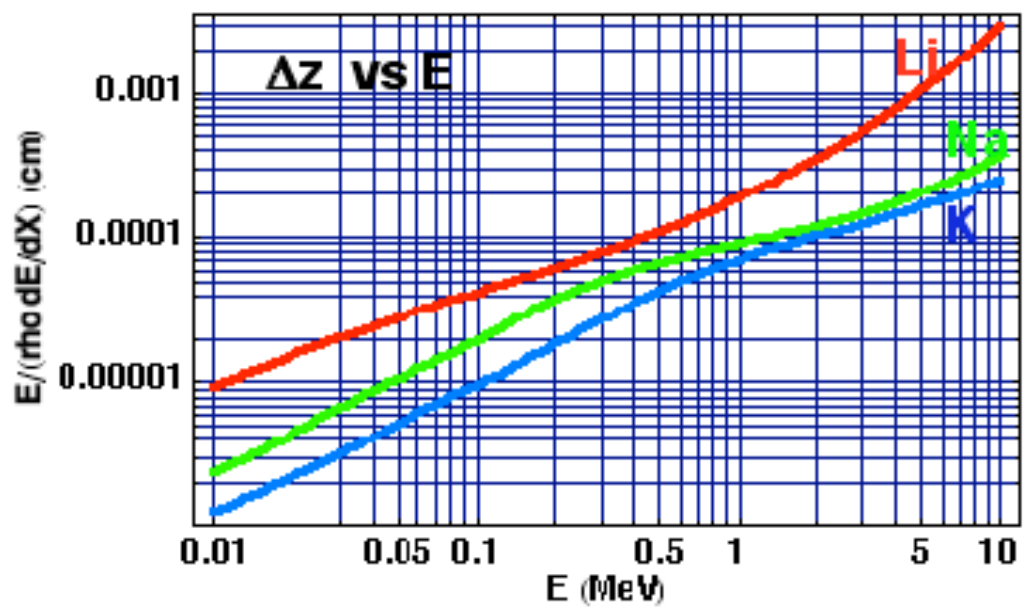

Figure 1.2 Range in $\mathrm{cm}(\mathrm{E} /(\rho \mathrm{dE} / \mathrm{dX}))$ as a function of ion energy in solid aluminum for three different ions $(\mathrm{K}, \mathrm{Na}$, and $\mathrm{Li})$ for energies that include the range of current and near-term future experiments (data obtained from the SRIM code).

III. Analytic theory using ideal gas equation of state 
The continuity equation for the mass density $\rho$, fluid velocity $v$, evolving in time $t$ and longitudinal coordinate $z$ may be written:

$\frac{\partial \rho}{\partial t}+\frac{\partial \rho v}{\partial z}=0$

The momentum equation can be written:

$\frac{\partial v}{\partial t}+v \frac{\partial v}{\partial z}=-\frac{1}{\rho} \frac{\partial p}{\partial z}$

For an adiabatic change in the material the pressure satisfies,

$p=K \rho^{\gamma}$

Here $\mathrm{K}$ is a constant $\left(=\mathrm{p}_{0} / \rho_{0}{ }^{\gamma}\right.$, where subscript 0 indicates initial values). The sound speed in the medium is give by: $c_{\mathrm{s}}^{2} \equiv \gamma P / \rho$. A similarity solution is found in the variable $z / c_{\mathrm{s} 0} t$ for the evolution of a rarefaction wave which propagates into an initial uniform medium (for $\mathrm{z}>0$ ) with initial pressure $\mathrm{p}_{0}$, density $\rho_{0}$, and sound speed $\mathrm{c}_{\mathrm{s} 0}$, and expands into what is initially a vacuum for $\mathrm{z}<0$. The solution (the so-called simple wave solution) in the region $-2 /(\gamma-1)<z / c_{\mathrm{s} 0} t<1$ is given by [2]:

$$
\begin{aligned}
& \frac{v}{c_{s 0}}=\left(\frac{2}{\gamma+1}\right)\left(\frac{z}{c_{s 0} t}-1\right) \\
& \frac{c_{s}}{c_{s 0}}=\left(\frac{\gamma-1}{\gamma+1}\right)\left(\frac{z}{c_{s 0} t}\right)+\frac{2}{\gamma+1} \\
& \frac{\rho}{\rho_{0}}=\left(\frac{c_{s}}{c_{s 0}}\right)^{2 /(\gamma-1)} ; \quad \frac{T}{T_{0}}=\left(\frac{c_{s}}{c_{s 0}}\right)^{2}
\end{aligned}
$$

Consider a slab of finite width $2 L$. In that case a rarefaction wave will propagate into the medium at speed $\mathrm{c}_{\mathrm{s} 0}$ from both edges of the slab $(z=0$ and $z=2 L)$. Let $\tau=c_{s 0} t / L$ and $\zeta$ $=z / L$. After $\tau=1$, the simple waves will collide and there will be a growing region (the 
non-simple wave region) not governed by eqs. (8) to (10).

The boundary between the simple waves and the non-simple waves is given [2] by:

$$
\zeta_{\text {boundary }}=\frac{-2}{(\gamma-1)} \tau+\left(\frac{\gamma+1}{\gamma-1}\right) \tau^{\frac{3-\gamma}{\gamma+1}}
$$

In ref.[2] it ishown how eqs. (5) - (7) may be transformed to a single second order partial differential equation for the transformed variable $\chi$, expressed as a function of the velocity $v$ and the enthalpy $w=c_{\mathrm{s}}^{2} /(\gamma-1)$ :

$$
\frac{2}{2 n+1} w \frac{\partial^{2} \chi}{\partial w^{2}}-\frac{\partial^{2} \chi}{\partial v^{2}}+\frac{\partial \chi}{\partial w}=0
$$

What are normally the independent variables $z$ and $t$ may be generated from:

$$
t=\partial \chi / \partial w ; \quad z=v \partial \chi / \partial w-\partial \chi / \partial v
$$

The general solution is [2]:

$\chi=\left(\frac{\partial}{c_{s} \partial c_{s}}\right)^{n-1}\left(\frac{1}{c_{s}} F_{1}\left(c_{s}+\frac{v}{2 n+1}\right)+\frac{1}{c_{s}} F_{2}\left(c_{s}-\frac{v}{2 n+1}\right)\right)$

Here $F_{1}$ and $F_{2}$ are arbitrary functions, and the solution is valid for positive integral values of $n$, where $n=(3-\gamma) /(2(\gamma-1))$.

The particular solution with $v=0$ at $z / L=1$, and $\chi=0$ along boundary curve [2],

$$
\chi=\frac{L(2 n+1)}{2^{n} n !}\left(\frac{\partial}{c_{s} \partial c_{s}}\right)^{n-1}\left(\frac{1}{c_{s}}\left[\left(c_{s}-\frac{v}{2 n+1}\right) 2+c_{s 0}^{2}\right]^{n}\right)
$$

So for a given value of $n$ one may calculate $\chi$, then take derivatives with respect to $c_{\mathrm{s}}$ and $v$ to find $t\left(c_{\mathrm{s}}, v\right)$ and $z\left(c_{\mathrm{s}}, v\right)$ and invert if possible. For example, for $\gamma=5 / 3,(n=1$, corresponding to a perfect gas with 3 degrees of freedom), the solution becomes: $\chi=L\left(9 c_{\mathrm{s}}^{2}-9 c_{\mathrm{s} 0}^{2}-6 c_{\mathrm{s}} v+v^{2}\right) /\left(6 \mathrm{c}_{\mathrm{s}}\right) ; t=L\left(9 c_{\mathrm{s}}^{2}+9 c_{\mathrm{s} 0}{ }^{2}-v^{2}\right) ;$ and $z=L\left(18 c_{\mathrm{s}}^{3}+3 c_{\mathrm{s}}^{2} v+9 c_{\mathrm{s} 0}{ }^{2} v-v^{3}\right) /\left(18 c_{\mathrm{s}}^{3}\right)$. From observation of the exact solution, we find that in the non-simple region the velocity is nearly linear starting from zero at the center of the slab $(\xi=1)$ to the value 
$v=v\left(\zeta=\zeta_{\text {boundary }}\right)$ on the boundary between the simple and non-simple wave. So an approximate solution $v_{\text {approx }} / c_{s 0}$ for the velocity in this region $\left(\zeta_{\text {boundary }}<\zeta<1\right.$, and $\left.\tau>1\right)$ is given by:

$\frac{v_{\text {approx }}}{c_{s 0}}=\frac{-2}{\gamma-1}\left(1-\tau^{\frac{2(1-\gamma)}{\gamma+1}}\right)\left(\frac{\zeta-1}{\zeta_{\text {boundary }}-1}\right)$

Similarly, the density in the non-simple wave region is observed to be nearly parabolic from center to the edge. Using conservation of total mass, and using the value of $\rho / \rho_{0}$ on the boundary $\zeta=\zeta_{\text {boundary }}$ it is easy to calculate the approximate density in the non-simple region $\left(\zeta_{\text {boundary }}<\zeta<1\right)$ :

$r_{\text {approx }} \equiv \frac{\rho}{\rho_{0}}=\left(r_{\max }-\tau^{\frac{-4}{\gamma+1}}\right)\left(1-\left(\frac{\zeta-1}{\zeta_{\text {boundary }}-1}\right)^{2}\right)+\tau^{\frac{-4}{\gamma+1}}$

where $r_{\max }$ is the value of $\rho / \rho_{0}$ at the center of slab $(\zeta=1)$ and is given by:

$r_{\max }=\frac{3}{2} \frac{\left(1-\tau^{-1}-\frac{1}{3} \tau^{-4 /(\gamma+1)}\left(1-\zeta_{\text {boundary }}\right)\right)}{\left(1-\zeta_{\text {boundary }}\right)}$

Thus, the simple wave solution (eqs. (8)- (10)), together with either the exact formulas (eq. (15)) or the approximate formulas in the non-simple wave region (eqs. (16) - (18)) give the complete solution to the evolution of a one-dimensional slab heated instantaneously to some temperature $\mathrm{T}_{0}$ assuming a perfect gas EOS. (The solutions are for $\zeta<1$; the $\zeta>1$ solution is obviously a mirror image of the $\zeta<1$ solution). The approximate formulas are more tractable and can be useful for understanding the scaling of the central part of the slab (for example), and for non-integer values of $n$. This ideal solution for a perfect gas can be used as a rough starting guide for interpretation of more complicated situations in which there is a non-ideal equation of state or deposition is over a finite time, and when the deposition is not entirely uniform. The main features of the 
solution are the expansion of the material outward at a velocity $v= \pm 2 c_{\mathrm{s} 0} /(\gamma-1)$, a

rarefaction wave propagating inward at velocity $c_{\mathrm{s} 0}$, and a decreasing central density (and temperature) for times after the rarefaction wave has reached the center of the slab $(\tau=1)$.

\section{Simulation results under instantaneous heating approximation}

To go beyond the analytic results in section III, requires the use of simulation codes. Two codes have been employed in this study. The code HYDRA [12] is a 3D radiation/hydrodynamics code used primarily for ICF simulations, and has been employed here for target hydrodynamics. The 1D hydrodynamics code DPC (written by Richard More), uses an equation of state specifically for the Warm Dense Matter regime based on the Saha equation using energy levels of neutral atoms, melting temperature and latent heat of the material studied [13]. DPC has also been extensively employed for hydrodynamics calculations of the target. Since the beam radius is of order $1 \mathrm{~mm}$, and the thickness of the target is of order a few microns for a solid, to a few hundred microns for a $1 \%$ foam, the $1 \mathrm{D}$ code should accurately represent the longitudinal physics at the center of the target. DPC uses a Maxwell construction for the EOS [13]. In the Maxwell construction, an isotherm of the EOS that has a region in $\rho$ that is dynamically unstable in the two phase regime $(\partial P / \partial \rho<0)$, is replaced by an isotherm with a region of constant pressure as a function of density, that bridges the liquid vapor transition. This construction yields the equilibrium value of pressure, and yields numerically more stable solutions, but does not resolve the material into bubbles and droplets. HYDRA has been employed using two different equations of state, QEOS [14] and LEOS, which employs a tabular equations of state. QEOS uses a Thomas Fermi model for the electron EOS and 
uses a modified Cowan model for the ion EOS. QEOS does not use the Maxwell construction, but LEOS allows use of either the Maxwellian or non-Maxwellian construction.

Recently [15], has used DPC to study the expansion of a tin foil, initially in a liquid state, but due to expansion of the foil into the two phase-regime, where liquids and vapors coexist. Figure 4.1, shows the results. Plateaus in densities and temperature are apparent in the spatial profile after finite times, as the material undergoes a phase transition. When HYDRA is run without using QEOS without the maxwell construction evidence for the plateaus remain at roughly the same longitudinal position (see figs. 4.2 and 4.3), although oscillations in density are apparent as the code tries to make bubbles (vapor density) and droplets (liquid density) in the two phase region. The spatial zoning is likely not fully resolving the droplets and bubble formation and the code does not include surface tension effects, so the limits to the accuracy of the simulation should be kept in mind.
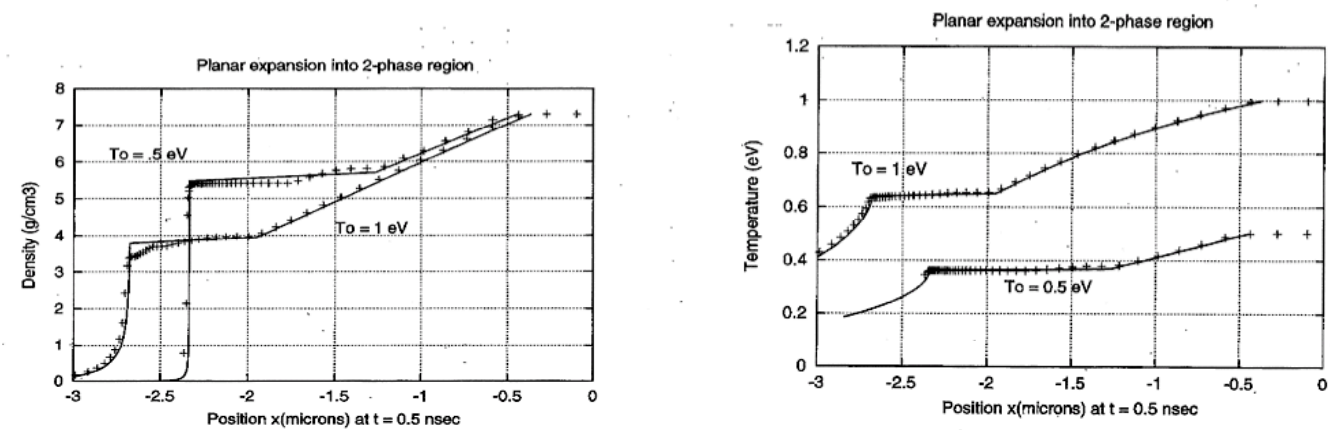

Figure 4.1. Snapshot of tin foil, with initial temperature $\mathrm{T}_{0}$ of 0.5 and $1.0 \mathrm{eV}$ after $0.5 \mathrm{~ns}$. The surface of the foil was initially at -2 microns in this DPC simulation. (Left: density vs. position. ; Right: Temperature) (from ref. [15].) 

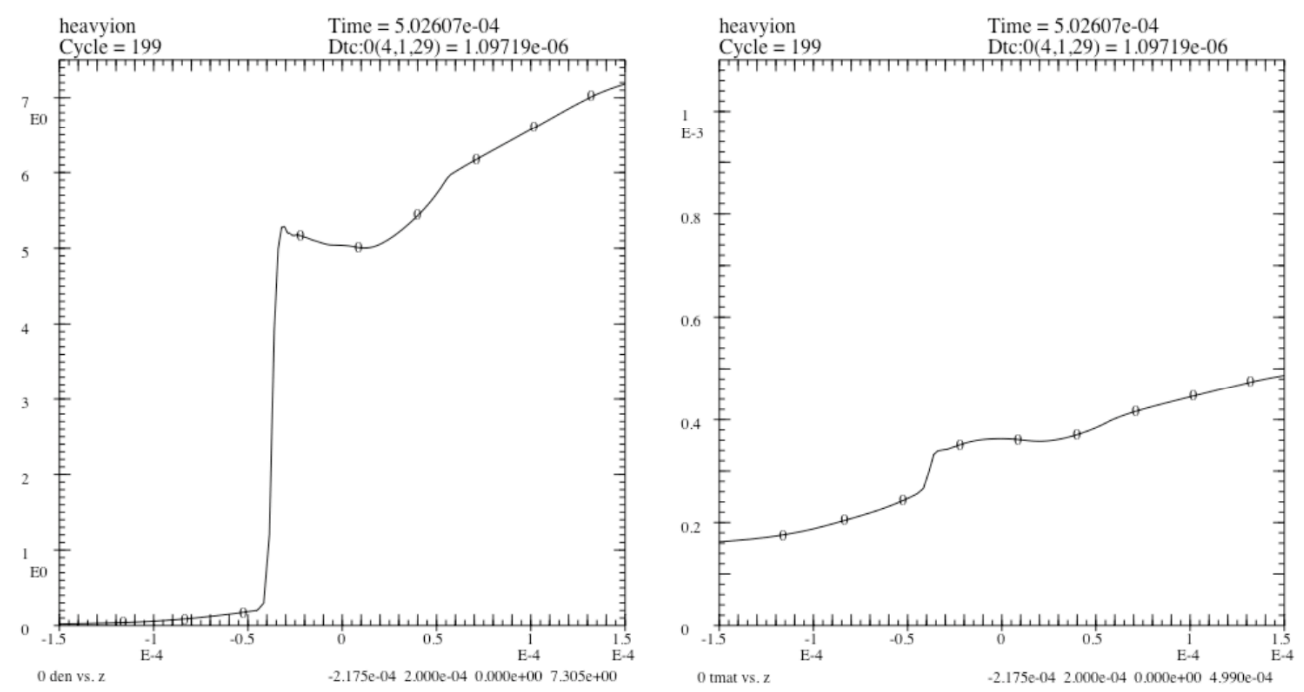

Figure 4.2. Snapshot of tin foil, with initial temperature $\mathrm{T}_{0}$ of $0.5 \mathrm{eV}$ after $0.5 \mathrm{~ns}$. The surface of the foil was initially at 0 microns in this HYDRA/QEOS simulation. (Left: density vs. position. Right: Temperature.)
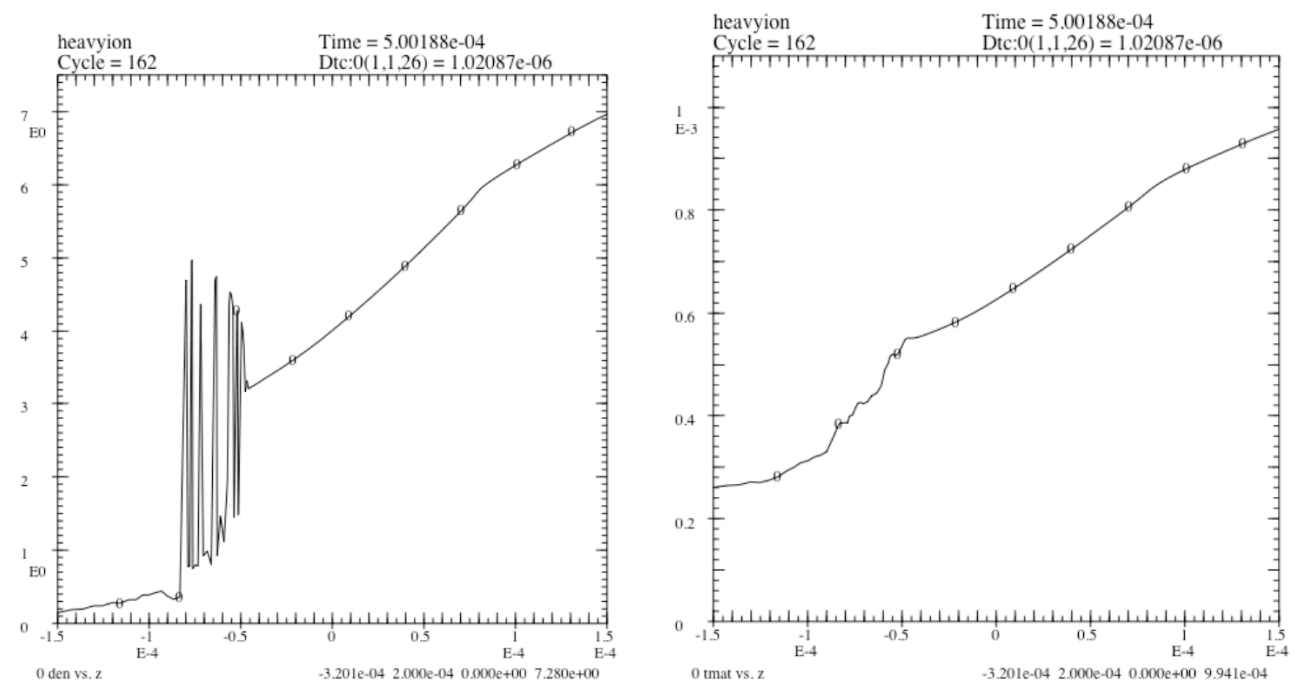

Figure 4.3. Snapshot of tin foil, with initial temperature $\mathrm{T}_{0}$ of $1.0 \mathrm{eV}$ after $0.5 \mathrm{~ns}$. The surface of the foil was initially at 0 microns in this HYDRA/QEOS simulation. (Left: density vs. position. Right: Temperature.)

\section{Parametric studies for NDCX II}


To understand the general behaviour of targets in the context of a particular experiment, we have chosen a set of ion beam and target parameters similar to those proposed for the NDCX II experiment proposed by the HIFS VNL [1]. In particular, we have modeled the dynamics of solid aluminum targets that have been heated by a $2.8 \mathrm{MeV} \mathrm{Li}^{+}$ion beam, one possible option for NDCX II. (2.8 MeV corresponds to an energy $50 \%$ higher than the Bragg peak energy, resulting in an approximately $5 \%$ variation in $d E / d X$, if a foil thickness is chosen such that the beam exits the foil at approximately $0.93 \mathrm{MeV}$ or half the energy at the Bragg peak). Other parameters for the "nominal" case included an ion fluence per unit area $F$ impinging on the target of $30 \mathrm{~J} / \mathrm{cm}^{2}$ and and an initial $d E / d X$ of $2.052 \mathrm{MeV}-\mathrm{cm}^{2} / \mathrm{mg}$ (as estimated by the SRIM code). The energy absorbed per mass $=F$ $d E / d X / E=21.986 \mathrm{Joules} / \mathrm{mg}=2.2 \times 10^{4} \mathrm{Joules} / \mathrm{g}=6.2 \mathrm{eV} /$ atom. Thus, the nominal target temperature, assuming a specific heat of $3 \mathrm{eV} /(\mathrm{eV}$-atom) would be approximately 2 $\mathrm{eV}$. The assumed pulse duration was $1 \mathrm{~ns}$, full width in a parabolic pulse. The nominal target was solid Aluminum $\left(A=26.98\right.$, density $\left.=2.7 \mathrm{grams} / \mathrm{cm}^{3}\right)$, with a nominal thickness of 3.5 microns. The pulse duration, thickness and fluence were varied, and runs were made in the two simulation codes described above, DPC and HYDRA.

Figures 5.1 and 5.2 show the temporal and spatial results of the nominal case from DPC. Figure 5.3 shows a comparison between DPC results and Hydra results using QEOS, of the evolution of the central temperature of the target. Detailed differences arising from different assumptions about the equation of state are apparent. Finally, some of the systematic variations of maximum central pressure, maximum central temperature and maximum surface velocity with deposition energy per target mass $(F d E / d X / E)$ are shown in figures $5.4,5.5$, and 5.6 for various target thicknesses. 
Some observations from can be made. The maximum central pressure achieved depends sensitively on the foil thickness (as well as the energy deposition). This is because different thicknesses allow the rarefaction wave to reach the center of the foil before all of the energy from the ion beam has been deposited. The pressure is most sensitive because it depends on both density and temperature decreases. The temperature also is reduced for thinner foils because of the cooling from expansion, but the ion deposition continues to increase the central temperature after the rarefaction wave has reached the center. The expansion velocity is the least sensitive to the foil thickness, perhaps because it reflects an integrated effect of the energy deposited. We should note that for the solution of the instantaneously heated perfect gas foil described in section III, we may write the energy density per mass $\varepsilon=c_{\mathrm{s} 0}{ }^{2} /(\gamma(\gamma-1))$, so that the maximum expansion velocity of the outward material can be expressed $v=\left(4 \gamma /(\gamma-1)^{1 / 2} \varepsilon^{1 / 2}\right.$. So for this case the ratio of the velocity to the energy deposition depends only on the equation of state (i.e. $\gamma$ in this model) which suggests that measurements of expansion velocity, will be useful in discriminating equations of state. 
Temperature $(\mathrm{eV})$

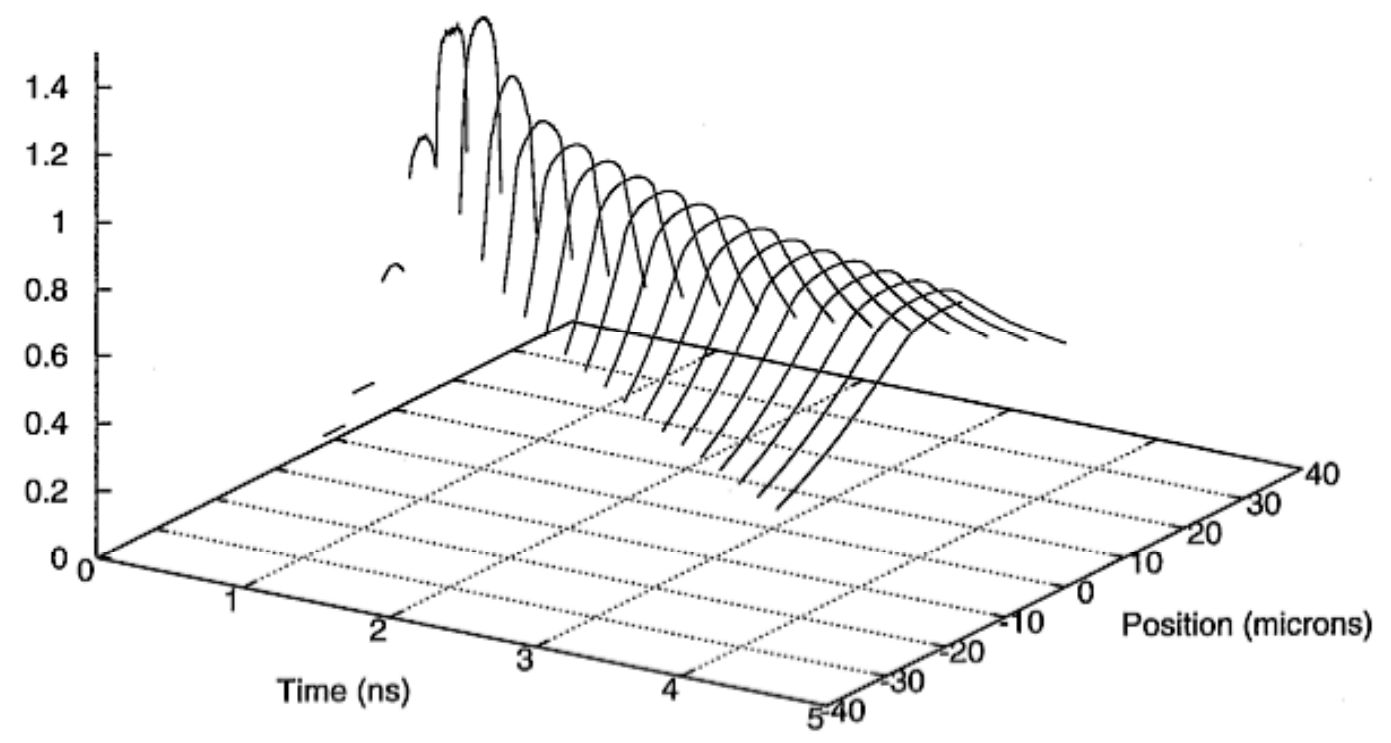

Figure 5.1 Evolution of the target temperature as a function of time and position for the nominal Li NDCX II parameters using the DPC code over the first $5 \mathrm{~ns}$. The foil is initially $3.5 \mu$ thick (centered at $z=0$ ) and by approximately 4 ns has expanded to cover 20-30 $\mu$. 


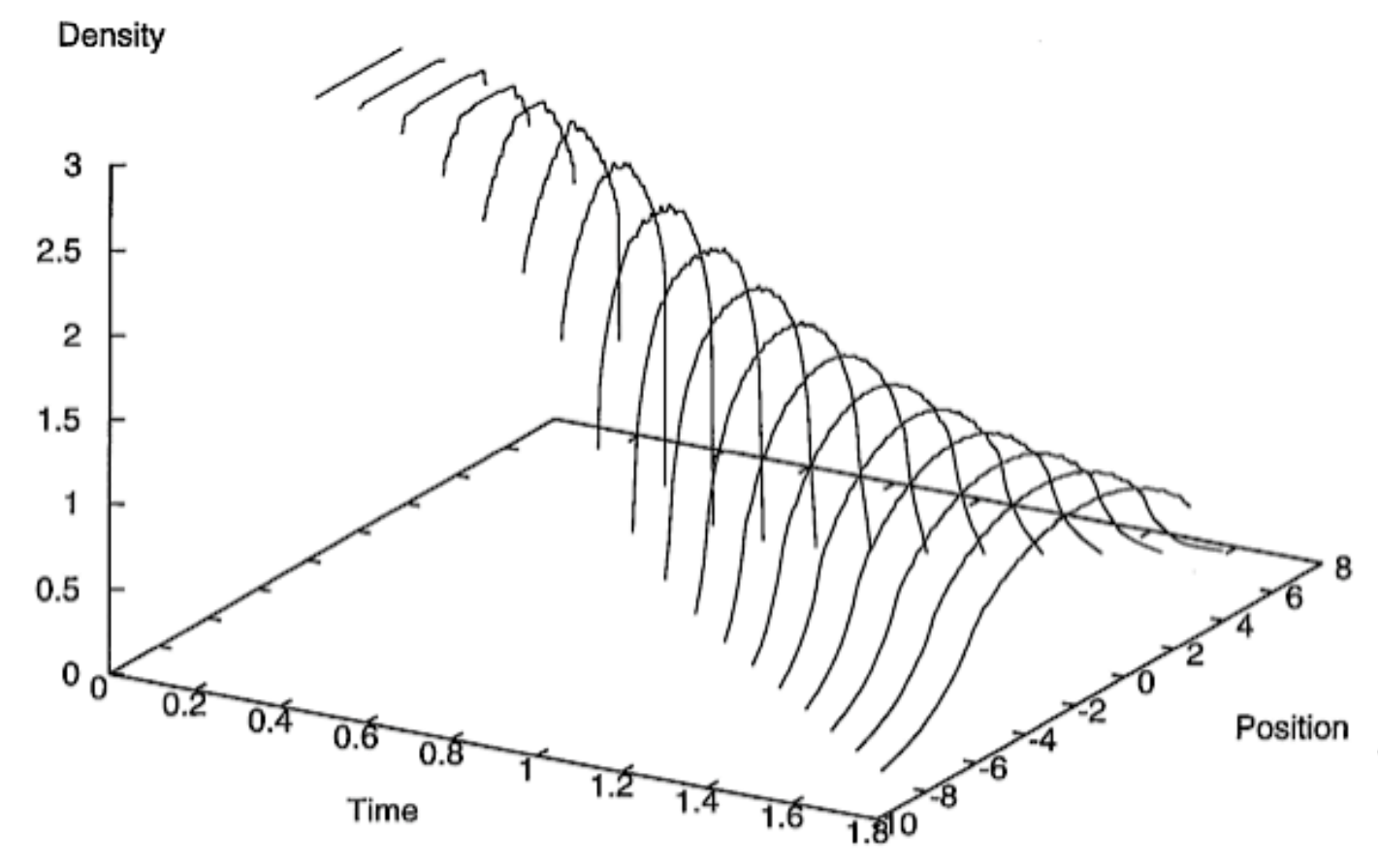

Figure 5.2 Evolution of the target density as a function of time and position for the nominal Li NDCX II parameters using the DPC code (for the first $1.8 \mathrm{~ns}$ ).
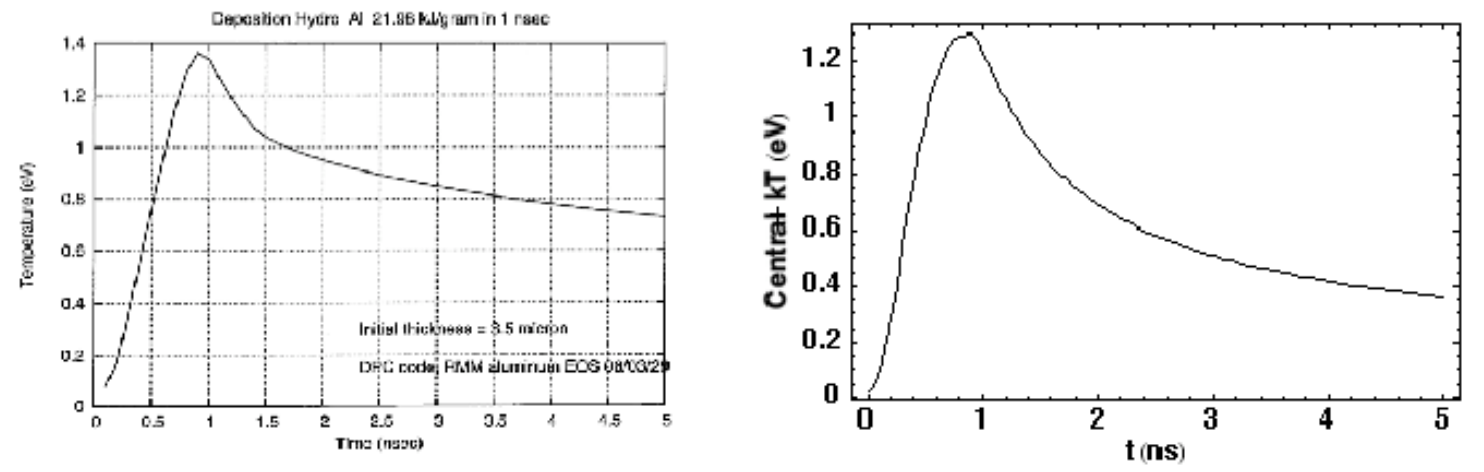

Figure 5.3 Comparison of the evolution of the central temperature of the nominal case using DPC (left) and Hydra (right). The change in slope at about $1.5 \mathrm{~ns}$ in the DPC result has been found to be associated with the entrance into the two-phase regime. 

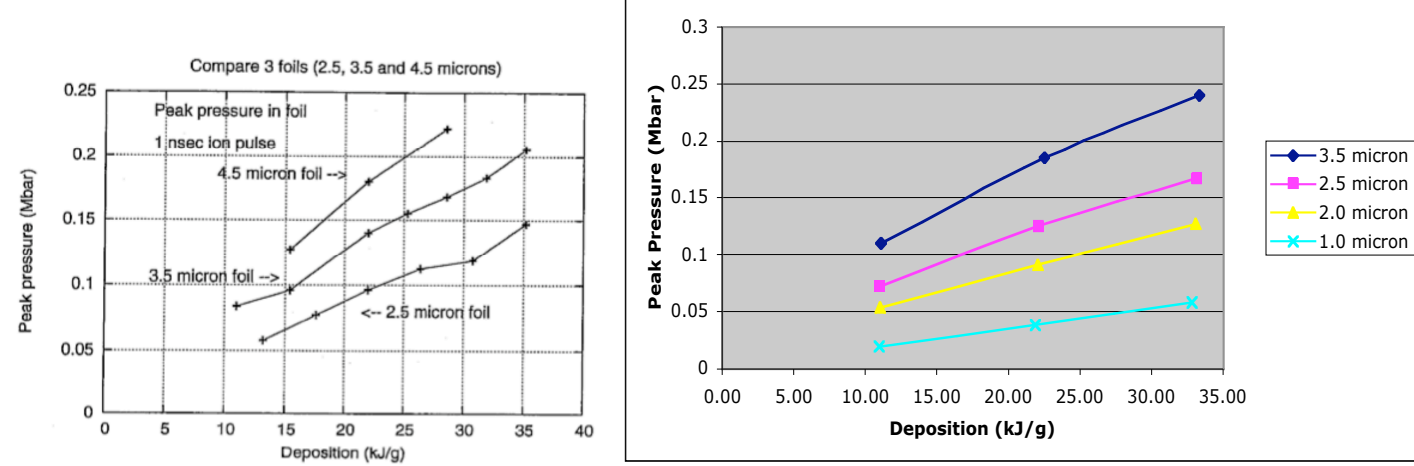

Figure 5.4 Peak pressure vs. energy deposition for three foil thickness (see text for parameters) using the DPC code (left) and four foil thickness using HYDRA/QEOS.
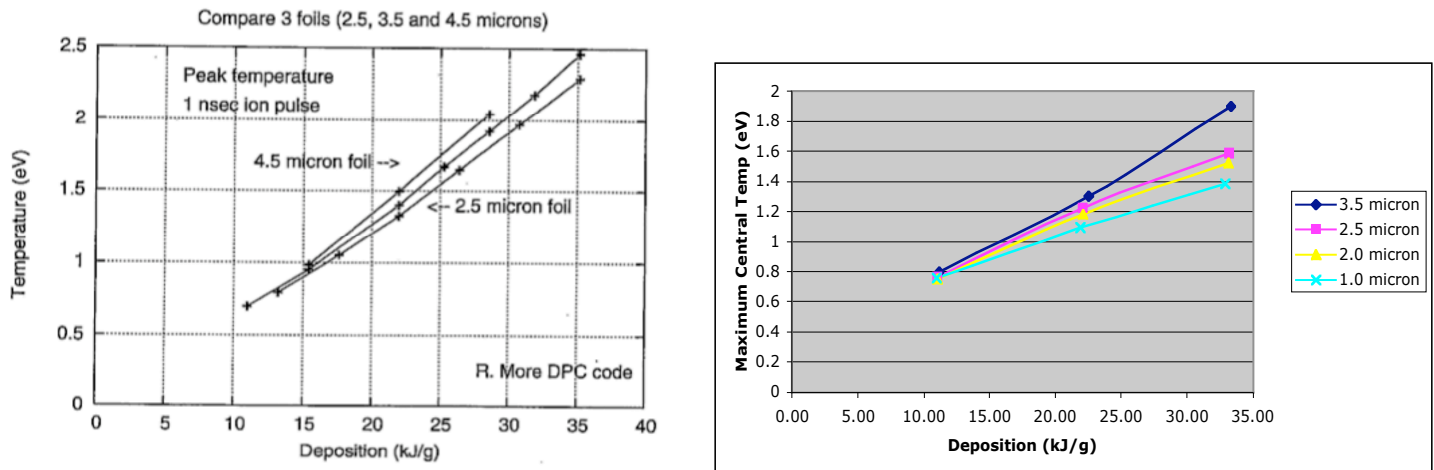

Figure 5.5 Peak temperature vs. energy deposition for three foil thickness (see text for parameters) using the DPC code (left) and four foil thickness using HYDRA/QEOS.
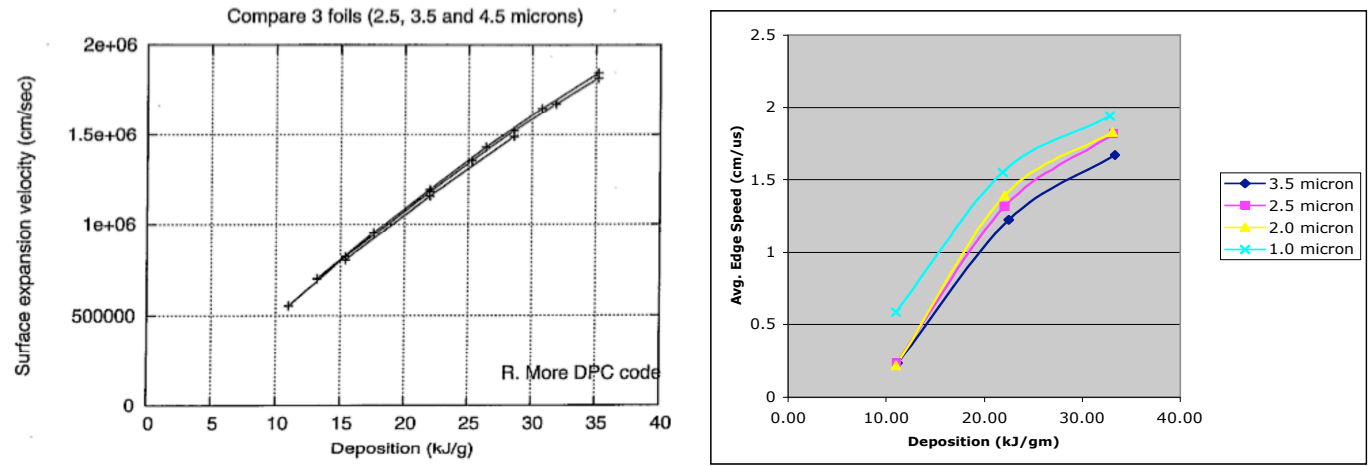
Figure 5.6 Release velocity vs. energy deposition vs. for three foil thicknesses (see text for parameters) using the DPC code (left) and four foil thickness using HYDRA/QEOS.

\section{Capturing the physics of Bubbles and Droplets}

As discussed above, neither DPC nor HYDRA captures the detailed physics of droplets or bubbles, which may be important in understanding the results of WDM experiments using ion beams. One deficiency is the lack of surface tension effects in either code.

We have begun to estimate the maximum size of droplets that would be created in the transition of the expanding foil from liquid to gas. In one estimate, the force on a droplet from the viscosity of the expanding gas tending to stretch the droplet will overcome the inward force of the surface tension tension if the radius of the droplet is sufficiently large. This yields a maximum droplet radius given by [16]:

$x=\sigma /(\mu d v / d x)$

This corresponds to a capillary number $(\equiv \mu \nu / \sigma)$ of order unity at the maximum radius. Here $\sigma$ is the coefficient of surface tension, $\mu$ is the viscosity and $d v / d x$ is the velocity gradient of the underlying expanding medium. Another estimate balances the dynamic pressure of an expanding droplet to the inward force of the surface tension. This yields a maximum droplet radius given by:

$x=\left(\sigma / \rho(d v / d x)^{2}\right)^{1 / 3}$

Here $\rho$ is the liquid droplet density. This corresponds to a weber number $\left(\equiv \rho \mathrm{xv}^{2} / \sigma\right)$ of order unity at the maximum radius. For typical numbers at a time when the material is in the two phase regime, $\left(d v / d x=10^{6} \mathrm{~cm} / \mathrm{s}, \sigma=100\right.$ dyne $/ \mathrm{cm}, \mu=5 \times 10^{-3} \mathrm{~g} / \mathrm{cm}-\mathrm{s}, \rho=1 \mathrm{~g} / \mathrm{cm}^{3}$, 
$v_{\mathrm{th}}=5 \times 10^{5} \mathrm{~cm} / \mathrm{s}$ ) we find maximum droplet radii of order 0.1 micron. Further

calculations including evaporation and condensation rates, and accurate estimations of $\sigma$ and $\mu$ are beginning to yield physical aspects of the droplets and bubbles, which may be useful as these effects are incorporated into the simulation codes.

\section{Summary}

We have carried out both analysis and and a variety of simulations for understanding the physics of ion beam heated target foils, in order to provide insight into planned future experiments using ion beams to heat material to warm dense matter conditions. Many issues still remain to be explored in more detail to refine our understanding of these experiments. Among these issues are foam homogenization, hydrodynamics through the two-phase region, effects of velocity spread and straggling of the ion beam, and acceptable levels of preheat. The ultimate goal of our simulation studies is to determine what observables will be most sensitive to allow minimization of the uncertainties in equation of state and other material quantities for a wide range of substances and over a large range in the density and temperature parameter space.

\section{References:}

[1] B.G. Logan, et al, "Recent U.S. advances in ion-beam-driven high energy density physics and heavy ion fusion," Nucl. Instr. and Meth. Phys. Res., these proceedings. [2] L.D. Landau and E.M. Lifshitz, "Fluid Mechanics," Pergamon Press (chapter 10) (1959).

[3] B.G. Logan, R.C.Davidson, J.J. Barnard, R. Lee, "A unique U.S. approach for accelerator driven Warm Dense Matter Research -- Preliminary Report," Proceedings of 
the Workshop on Accelerator-Driven High Energy Density Physics, Appendix A1, held at LBNL, October 26-29, 2004, LBNL-57518, UCRL-PROC-212000, (2005)

(http://hifweb.lbl.gov/public/hedpworkshop/toc.html)

[4] L. R. Grisham, Moderate Energy Ions for High Energy Density Physics Experiments, Physics of Plasmas, 11, 5727 (2004).

[5] F.M. Bieniosek, J. Barnard, M.A. Leitner, A.W. Molvik, R. More, P.K. Roy, "Diagnostics for near term WDM experiments", Nucl. Instr. and Meth. Phys. Res., these proceedings.

[6] P. K. Roy, S. S. Yu, S. Eylon, E. Henestroza, A. Anders, E. P. Gilson, F. M.

Bieniosek, W. G. Greenway, B. G. Logan, W. L. Waldron, D. B. Shuman, D. L.

Vanecek, D. R. Welch, D. V. Rose, C. Thoma, R. C. Davidson, P. C. Efthimion, I.

Kaganovich, A. B. Sefkow, and W. M. Sharp, Nucl. Instr. Meth. Phys. Res. A544 (2005) 225.

[7] J. J. Barnard, R. J. Briggs, D.A. Callahan, R.C. Davidson, A. Friedman, L. Grisham, E. P. Lee, R. W. Lee, B. G. Logan, C. L. Olson, D. V. Rose, P. Santhanam, A. M. Sessler, J. W. Staples, M. Tabak, D. R. Welch, J. S. Wurtele, S. S. Yu., " Accelerator and ion beam tradeoffs for studies of Warm Dense Matter," Proceedings of the Particle Accelerator Conference, May 2005, Knoxville, TN, RPA039 (2005).

[8] Roy, P.K.; Yu, S.S.; Henestroza, E.; Anders, A.; Bieniosek, F.M.; Coleman, J.; Eylon, S.; Greenway, W. G.; Leitner, M.; Logan, B.G.; Waldron, W.L.; Welch, D.R.; Thoma, C.; Sefkow, A.B.; Gilson, E.P.; Efthimion, P.C.; Davidson, Phys. Rev. Lett. 95 (2005) 234801. 
[9] P.K. Roy, S.S. Yu, E. Henestroza, A. Anders, D. Baca, F.M. Bieniosek, J. Coleman, R.C. Davidson, P.C. Efthimion, S. Eylon, E.P. Gilson, W.G. Greenway, I. Kaganovich, M. Leitner, B.G. Logan, A.B. Sefkow, P. Seidl, Nucl. Instr. and Meth. Phys. Res., these proceedings.

[10] A. Sefkow, R.C. Davidson, I.D. Kaganovich, E.P. Gilson, P.K. Roy, S.S. Yu, P.A. Seidl, D.R. Welch D.V. Rose, J.J. Barnard, “Optimized Simultaneous Transverse and Longitudinal Focusing of Intense Ion Beam Pulses for Warm Dense Matter Applications", Nucl. Instr. and Meth. Phys. Res., these proceedings.

[11] C. Thoma, W.L. Waldron, D.R. Welch, "Neutralized Drift Compression Experiments (NDCX) with a High Intensity Ion Beam”, Nucl. Instr. and Meth. Phys. Res., these proceedings.

[12] M. M. Marinak, G. D. Kerbel, N. A. Gentile, O. Jones, D. Munro, S. Pollaine, T. R. Dittrich, and S. W. Haan, Phys. Plasmas 8, 2275 (2001).

[13] R. More, H. Yoneda and H. Morikami, JQSRT 99, 409 (2006).

[14] R. More, K.H. Warren, D.A. Young, G. B. Zimmerman, "A new quotidian equation of state for hot dense matter", Phys. Fluids, 31, 3062 (1988).

[15] R. More, Kato, and H. Yoneda, preprint.

[16] J. Armijo, master's internship report, Ecole Normale Superieure, Paris, 2006, (in preparation).

[17] D. R. Welch, D.V. Rose, A.B. Sefkow, "Avoiding preheat in a Warm Dense Matter Experiment", Workshop on Accelerator Driven Warm Dense Matter Physics, Pleasanton, CA, February 2006. http://hifweb.lbl.gov/public/AcceleratorWDM/TableOfContents.html This work was performed under the auspices of the U.S. Department of Energy by University of California, Lawrence Livermore National Laboratory under Contract W-7405-Eng-48. 